\title{
Experience in Online activity to introduce clinical trial design for undergraduate Students of School of Pharmacy, Universidad de Buenos Aires
}

\author{
Susana Gorzalczany, Cecilia Kravetz, Angeles Yrbas, Maria Silvia Viola \\ Universidad de Buenos Aires, School of Pharmacy and Biochemistry, Argentina
}

Training in the scientific method and research design will not only benefit pharmacy students, but will also provide students with critical thinking, analytical and lifelong learning skills, which will enable them to handle challenges and situations as they arise.

Taking into account that the use of technological tools on learning could improve teaching, the aim of this study was introduced different topics of clinical research to undergraduate students of School of Pharmacy in Universidad de Buenos Aires, Argentina using Moodle (Modular Object-Oriented Dynamic Learning) platform. The activity was conducted for the first time in 2017 academic year. Different strategies were used.

Firstly, it was used a Ted Talk related to this issue and the students had to comment on it in a discussion forum. Secondly, taking into account that movies can be a powerful tool for promoting a visualization of concepts, it was used Miss Ever s Boys movie in order to discuss ethical aspects in clinical research. In this phase, a questionnaire was included and it was focus mainly in bioethics principles. Afterwards, preclinical and clinical studies (Phases I, II, III and IV) published in journals of medical research was analyzed. This activity tried to introduce not only ethical and regulatory aspects of clinical research but other relevant conditions in this field, such as experimental design (primary and secondary endpoint, double blind, randomization, inclusion and exclusion criteria, etc.). Finally, the students must compile a glossary of useful terms and concepts related to clinical trial they should have learned during the course, using wiki tool from moodle platform. Also, a survey was included at the end of the activity in order to know student opinion.

Taking into consideration the importance of formative assessment and the use of information and communications technology in higher education, this study showed different options that the implementation of the Moodle platform provides. Also, the purpose of this initiative was to develop a range of skills and abilities in students to enhance their interest for clinical research by promoting group interaction and motivating them in order to improve their academic performance and comprehensive training. 